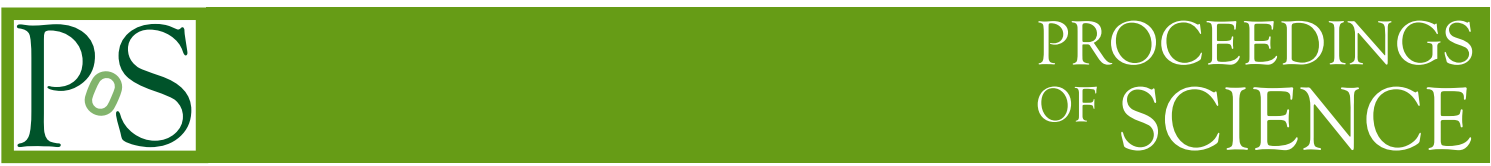

\title{
Discovery of Chromomagnetic Gluon Condensation
}

\author{
George Savvidy*† \\ Institute of Nuclear and Particle Physics \\ Demokritos National Research Centre \\ Agia Paraskevi, GR-15310 Athens, Greece \\ E-mail: savvidyr@inp.demokritos.gr
}

I reexamine the phenomena of the chromomagnetic gluon condensation in Yang-Mills theory. The extension of the Heisenberg-Euler Lagrangian to the Yang-Mills theory allows to predict the chromomagnetic gluon condensation. The energy density curve crosses the zero energy level of the perturbative vacuum state at nonzero angle and continuously enters into the negative energy density region. At the crossing point and further down the effective coupling constant is small and demonstrate that the true vacuum state of the Yang-Mills theory is below the perturbative vacuum state and is described by the nonzero chromomagnetic gluon condensate. The renormalisation group analyses allows to express the energy momentum tensor, its trace and the vacuum magnetic permeabilities in terms of effective coupling constant and Callan-Symanzik beta function. In the vacuum the energy-momentum tensor is proportional to the space-time metric and induces a negative cosmological constant.

Corfu Summer Institute 2019 "School and Workshops on Elementary Particle Physics and Gravity" (CORFU2019)

31 August - 25 September 2019

Corfu, Greece

\section{* Speaker.}

$\dagger$ Based on lectures at the Leipzig University in occasion of the 80 Years of Heisenberg-Euler Lagrangian 1936-2016, ITP, Leipzig, November 21, 2016 and 40 Years of Discovery of the Chromomagnetic Gluon Condensation 1977-2017 at the Ludwig-Maximilian University München, Arnold Sommerfeld Colloquium at Center for Theoretical Physics, April 18, 2018. 


\section{Effective Action in QED and QCD}

In this presentation I will reexamine the effective action in QED and QCD by using the perturbative loop expansion and renormalisation group equations and discuss the physical consequences which can be derived from their explicit expressions. The discovery of the chromomagnetic gluon condensation in Yang-Mills (YM) theory and the new results will be discussed and analysed [11, 12, 13, 14, 15, 15].

The Heisenberg-Euler effective Lagrangian in QED [1, 2, 3, 4, 5, 6] is a sum of the one loop diagrams with a vacuum electron-positron pair circulating in the loop and the gluons and quarks in case of QCD $[7,8,9,10,11,12,13,14,15]$. The effective action $\Gamma[A]$ has the following representation:

$\Gamma[A]=\int \mathcal{L} d x=\sum_{n} \int d x_{1} \ldots d x_{n} \Gamma_{\mu_{1} \ldots \mu_{n}}^{(n) a_{1} \ldots a_{n}}\left(x_{1}, \ldots, x_{n}\right) A_{\mu_{1}}^{a_{1}}\left(x_{1}\right) \ldots A_{\mu_{n}}^{a_{n}}\left(x_{n}\right)=S+W^{(1)}+\ldots$,

where $\mathcal{L}$ is the effective Lagrangian, $\Gamma^{(n)}$ is a one-particle irreducible (1PI) n-point vertex function, $A_{\mu}^{a}(x) \equiv\left\langle 0\left|\mathcal{A}_{\mu}^{a}(x)\right| 0\right\rangle$ is the vacuum expectation value of the field operator $\mathcal{A}_{\mu}^{a}$ and $W^{(n)}, n=1,2, .$. represent the terms of the loop expansion.

Considering the limit of massless electrons and quarks one can demonstrate that the proper time integral in the Heisenberg-Euler Lagrangian can be integrated explicitly [15] by using covariant renormalisation condition $[11,13,14]$

$$
\left.\frac{\partial \mathcal{L}}{\partial \mathcal{F}}\right|_{t=\frac{1}{2} \ln \left(\frac{2 e^{2}|\mathcal{F}|}{\mu^{4}}\right)=\mathcal{G}=0}=-1,
$$

where $\mathcal{F}=\frac{1}{4} G_{\mu \nu}^{a} G_{\mu \nu}^{a}$ is the Lorentz and gauge invariant form of the YM field strength tensor $G_{\mu \nu}^{a}$ and $\mu^{2}$ is the renormalisation scale parameter. In the massless limit the QED effective Lagrangian [15] has the exact logarithmic dependence as a function of the invariant $\mathcal{F}$ shown on Fig.1:

$$
\mathcal{L}_{e}=-\mathcal{F}+\frac{e^{2} \mathcal{F}}{24 \pi^{2}}\left[\ln \left(\frac{2 e^{2} \mathcal{F}}{\mu^{4}}\right)-1\right], \quad \mathcal{F}=\frac{\overrightarrow{\mathcal{H}}^{2}-\overrightarrow{\mathcal{E}}^{2}}{2}, \quad \mathcal{G}=\overrightarrow{\mathcal{E}} \overrightarrow{\mathcal{H}}=0
$$

where $\overrightarrow{\mathcal{H}}$ and $\overrightarrow{\mathcal{E}}$ are magnetic and electric fields. This expression should be compared with the one-loop effective Lagrangian in pure $\mathrm{SU}(\mathrm{N})$ gauge field theory, which has the form $[11,13]$ (see Fig.2):

$$
\mathcal{L}_{g}=-\mathcal{F}-\frac{11 N}{96 \pi^{2}} g^{2} \mathcal{F}\left(\ln \frac{2 g^{2} \mathcal{F}}{\mu^{4}}-1\right), \quad \mathcal{F}=\frac{\overrightarrow{\mathcal{H}}_{a}^{2}-\overrightarrow{\mathcal{E}}_{a}^{2}}{2}>0, \quad \mathcal{G}=\overrightarrow{\mathcal{E}}_{a} \overrightarrow{\mathcal{H}}_{a}=0 .
$$

From (1.3) it follows that the corresponding quark contribution considered in the chiral limit is

$$
\mathcal{L}_{q}=-\mathcal{F}+\frac{N_{f}}{48 \pi^{2}} g^{2} \mathcal{F}\left[\ln \left(\frac{2 g^{2} \mathcal{F}}{\mu^{4}}\right)-1\right]
$$

where $N_{f}$ is the number of quark flavours. 


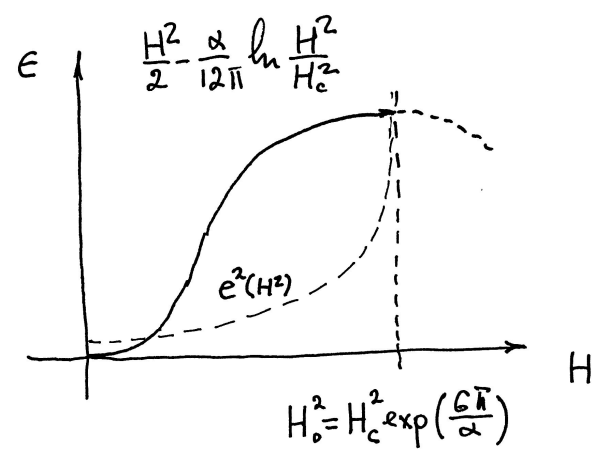

Figure 1: The graph shows the qualitative behaviour of the QED vacuum energy density $\epsilon\left(\overrightarrow{\mathcal{H}}^{2}\right)$ (1.12) and of the effective coupling constant $\bar{e}^{2}\left(\overrightarrow{\mathcal{H}}^{2}\right)(2.1)$ as the functions of magnetic field. The effective coupling constant is singular at $\overrightarrow{\mathcal{H}}_{0}{ }^{2}$, the "Moscow zero" [25, 26].

The effective Lagrangian technique allows to calculate the magnetic induction $\overrightarrow{\mathcal{B}}$ of the vacuum through the derivative of the effective Lagrangian [11]:

$$
\overrightarrow{\mathcal{B}}_{a}=-\frac{\partial \mathcal{L}}{\partial \overrightarrow{\mathcal{H}}_{a}}=\mu_{\text {vac }} \overrightarrow{\mathcal{H}}_{a}
$$

From (1.3), (1.4) and (1.5) it follows that in QED the vacuum responds to the background magnetic field as diamagnet and in QCD as paramagnet with the magnetic permeabilities of the following form [11]:

$$
\begin{array}{ll}
\mu_{Q E D}=1-\frac{e^{2}}{24 \pi^{2}} \log \left(\frac{e^{2} \overrightarrow{\mathcal{H}}^{2}}{\mu^{4}}\right)<1, & \text { diamagnetic, } \\
\mu_{Q C D}=1+\frac{g^{2}}{96 \pi^{2}}\left(11 N-2 N_{f}\right) \log \frac{g^{2} \overrightarrow{\mathcal{H}}_{a}^{2}}{\mu^{4}}>1, \quad \text { paramagnetic, } \quad N>\frac{2}{11} N_{f} .
\end{array}
$$

The diamagnetism of the QED vacuum (1.7) means that it repels the magnetic fields by forming induced magnetic field in the direction opposite to that of the applied magnetic field. This phenomenon is similar to the Landau orbital diamagnetism of free electron gas when the counteracting field is formed when the electron trajectories are curved due to the Lorentz force [17].

The paramagnetism of the QCD vacuum (1.8) means that it amplifies the applied chromomagnetic field by generating induced chromomagnetic field in the direction of the applied field. In QCD the large polarisation of the gluon spins is responsible for the amplification of the background field. This phenomenon is similar to the Pauli paramagnetism, an effect associated with the polarisation of the electron spins [16].

Using the effective Lagrangian approach one can calculate the quantum-mechanical corrections to the energy momentum tensor by using the formula derived by Schwinger [5]:

$$
T_{\mu \nu}=\left(F_{\mu \lambda} F_{\nu \lambda}-g_{\mu \nu} \frac{1}{4} F_{\lambda \rho}^{2}\right) \frac{\partial \mathcal{L}}{\partial \mathcal{F}}-g_{\mu \nu}\left(\mathcal{L}-\mathcal{F} \frac{\partial \mathcal{L}}{\partial \mathcal{F}}-\mathcal{G} \frac{\partial \mathcal{L}}{\partial \mathcal{G}}\right) .
$$


In case of the Heisenberg-Euler effective Lagrangian Schwinger presented the expression for the $T_{\mu \nu}$ in terms of the fine structure constant $\alpha=e^{2} / 4 \pi$ expansion:

$$
T_{\mu \nu}=T_{\mu \nu}^{M}\left(1-\frac{16}{45 m^{4}} \alpha^{2} \mathcal{F}\right)+g_{\mu \nu} \frac{2}{45 m^{4}} \alpha^{2}\left(4 \mathcal{F}^{2}+7 \mathcal{G}^{2}\right)+\ldots
$$

with its nonzero trace

$$
T=T_{\mu \mu}=\frac{8}{45 m^{4}} \alpha^{2}\left(4 \mathcal{F}^{2}+7 \mathcal{G}^{2}\right)+\ldots
$$

In massless QED using the one-loop expression (1.3) for $T_{\mu \nu}$ we can get

$$
T_{\mu \nu}=T_{\mu \nu}^{Q E D}\left[1-\frac{e^{2}}{24 \pi^{2}} \ln \frac{2 e^{2} \mathcal{F}}{\mu^{4}}\right]+g_{\mu \nu} \frac{e^{2}}{24 \pi^{2}} \mathcal{F}, \quad \mathcal{G}=0 .
$$

The $T_{\mu \nu}$ becomes proportional to the space-time metric tensor $g_{\mu \nu}$ at the extreme magnetic field $H_{0}^{2}=H_{c}^{2} \exp (6 \pi / \alpha)$ and therefore induces a positive effective cosmological constant (see Fig.1).

To calculate the energy momentum tensor $T_{\mu \nu}$ in pure $S U(N)$ YM theory one should use the expression (1.4). Considering the QCD in the limit of chiral fermions, one should also add the quark contribution (1.5) by using the substitution $11 N \rightarrow b=11 N-2 N_{f}$ :

$$
T_{\mu \nu}=T_{\mu \nu}^{Q C D}\left[1+\frac{b g^{2}}{96 \pi^{2}} \ln \frac{2 g^{2} \mathcal{F}}{\mu^{4}}\right]-g_{\mu \nu} \frac{b g^{2}}{96 \pi^{2}} \mathcal{F}, \quad \mathcal{G}=0
$$

It is worth to compare the expressions for the energy momentum tensors in QED (1.12) and QCD (1.13). They clearly stress the physical differences between the two gauge field theories, exposed in the form of opposite signs in front of the logarithmic terms and in the front of the anomalous contributions to the energy momentum tensor traces. The last terms are proportional to the metric tensor of the space time and naturally generate the effective contribution to the cosmological constant $[18,19,20]$. These contributions are well defined and are finite physical quantities in renormalisable gauge field theories. Both expressions for the energy momentum tensors allow directly analyse the structure and the physical properties of the vacuum in respective gauge field theories [27, 28, 29, 29, 30, 31, $32,33,34,35,36,37,38,39,40,41,45,46,47]$

Let us consider first the vacuum energy density $T_{00} \equiv \epsilon(\mathcal{F})$ which has in QCD the following form [13]:

$$
\epsilon(\mathcal{F})=\mathcal{F}+\frac{b g^{2}}{96 \pi^{2}} \mathcal{F}\left(\ln \frac{2 g^{2} \mathcal{F}}{\mu^{4}}-1\right)
$$

The energy density has its new minimum outside the perturbative vacuum state $G_{\mu \nu}^{2}(\langle A\rangle)=$ 0 , at Lorentz and renormalisation group invariant field strength which receives a nonzero contribution through the chromomagnetic gauge field condensate [13]

$$
\left\langle 2 g^{2} \mathcal{F}\right\rangle_{v a c}=\mu^{4} \exp \left(-\frac{96 \pi^{2}}{b g^{2}(\mu)}\right)=\Lambda_{Q C D}^{4},
$$

where $b=11 N-2 N_{f}$ and $\langle\ldots\rangle_{v a c}$ means the average over the vacuum gauge field orientations. The Lorentz invariant form of the effective action (1.4) suggests that there are many 


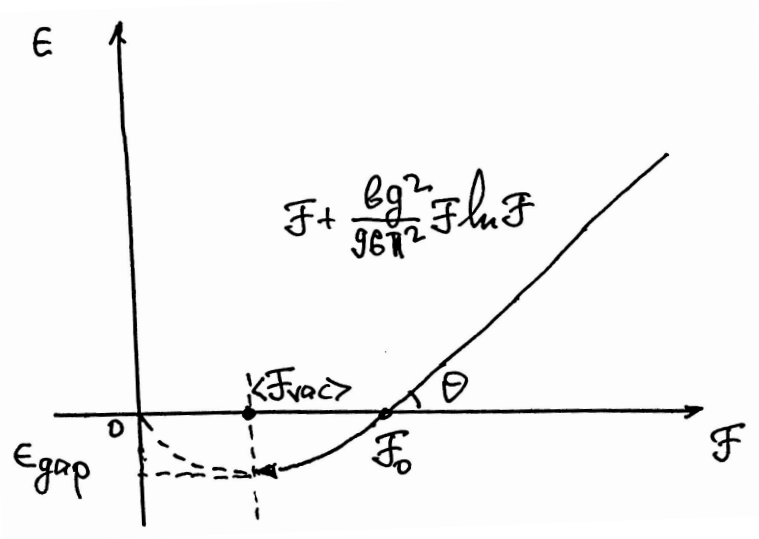

Figure 2: The graph shows the qualitative behaviour of the vacuum energy density $\epsilon(\mathcal{F})(1.14)$. At the intersection point $\mathcal{F}_{0}(2.11)$ the effective coupling constant is small (2.12) and the intersection angle $\theta$ is strictly positive (2.13). The energy density curve can be continuously extended from point $\mathcal{F}_{0}$ deep into the negative energy density region arbitrary close to the value of the vacuum condensate $\langle\mathcal{F}\rangle_{v a c}$ by considering a larger values of $N$ and keeping the t'Hooft coupling constant $g^{2} N$ fixed (2.14), (2.15). This proves that there is a nonzero energy gap $\epsilon_{g a p}>0$ between perturbative and true vacuum states. The vacuum is characterised by the nonzero value of the chromomagnetic field strength tensor (1.15), (2.7) and the energy density gap $\epsilon_{\text {gap }}(1.17)[13,27]$.

states which have the same energy density as the covariantly constant chromomagnetic field. In a series of articles [21, 22, 23, 24] the authors found and explored spatially homogeneous solutions of the YM equations which are invariant with respect to the Lorentz transformations and conveniently represent the gauge field fluctuations in the vacuum. The average $\langle\ldots\rangle_{\text {vac }}$ can be understood as average over these gauge field configurations. The condensate generates a dynamical symmetry breaking of the scaling invariance ${ }^{1}$ :

$$
\left\langle T_{\mu \mu}\right\rangle_{v a c}=-\frac{b}{48 \pi^{2}}\left\langle 2 g^{2} \mathcal{F}\right\rangle_{v a c}
$$

Substituting the vacuum field intensity (1.15) into the expression for the energy momentum tensor (1.13) one can get that in the vacuum the tensor $T_{\mu \nu}$ is proportional to the space-time metric $g_{\mu \nu}$ :

$$
\left\langle T_{\mu \nu}\right\rangle_{v a c}=-g_{\mu \nu} \frac{b}{96 \pi^{2}}\left\langle g^{2} \mathcal{F}\right\rangle_{v a c} .
$$

In this form the energy momentum tensor represents the relativistically invariant equation of the vacuum state $\epsilon_{v a c}=-P_{v a c}$ and uniquely characterises the vacuum $[18,19,20]$ with its negative energy density $\epsilon_{v a c}$. The vacuum energy momentum tensor (1.16) generates the effective cosmological constant $\Lambda_{\text {eff }}$

$$
R_{\mu \nu}-\frac{1}{2} g_{\mu \nu} R=g_{\mu \nu} \Lambda_{e f f}+\frac{8 \pi G}{c^{4}} T_{\mu \nu}=\frac{8 \pi G}{c^{4}}\left(\left\langle T_{\mu \nu}\right\rangle_{v a c}+T_{\mu \nu}\right)
$$

\footnotetext{
${ }^{1}$ The $\Lambda_{Q C D}$ is defined here through the covariant subtraction scheme (1.2). The relation with other renormalisation schemes was derived in [27].
} 
of the form:

$$
\epsilon_{v a c}=\frac{c^{4} \Lambda_{e f f}}{8 \pi G}=-\frac{b}{96 \pi^{2}}\left\langle g^{2} \mathcal{F}\right\rangle_{v a c}=-\frac{b}{192 \pi^{2}} \Lambda_{Q C D}^{4},
$$

where the chromomagnetic condensate $(1.15)$ is $\left\langle 2 g^{2} \mathcal{F}\right\rangle_{v a c}=\Lambda_{Q C D}^{4}$. The magnetic permeability (1.8) in the vacuum state (1.15) is equal to zero:

$$
\mu_{v a c}^{Q C D}=1+\frac{b g^{2}}{96 \pi^{2}} \log \frac{\left\langle 2 g^{2} \mathcal{F}\right\rangle_{v a c}}{\mu^{4}}=0 .
$$

The chromomagnetic condensate (1.15) is of order $\Lambda_{Q C D}^{4}$, and the vacuum energy density is negative and is about $\epsilon_{v a c} \approx-b 10^{-8} \mathrm{GeV}^{4}$. The value of the cosmological constant measured in the observation of the high-z Type Ia supernovae $[48,49,50,51]$ and by the Plank Collaboration [52, 53] $\epsilon_{\Lambda}=c^{4} \Lambda_{\text {obser }} / 8 \pi G \approx 10^{-47} \mathrm{GeV}^{4}$ is about 39 decimal places smaller and positive. The energy gap depends on a gauge group and a matter content, the $b$ parameter in beta function, as well as of the temperature of the universe [44]. At high temperatures the curve of the effective potential moves upward, the value of the chromomagnetic gluon condensate tends to zero, as well as the $\Lambda_{e f f}$, and the scaling invariance get restored. The phase transition is of the second-order [44]. In the article [54] the authors suggested a possible cancelation mechanism between chromomagnetic and its "mirror chromoelectric" condensates.

\section{Effective Lagrangian and Renormalisation Group}

It is useful to derive the expression of the effective Lagrangian by using the renormalisation group equation $[13,14]$. The solution of the renormalisation group equation in terms of effective coupling constant $\bar{g}(g, t)$, with the boundary condition $\bar{g}(g, 0)=g$, has the following form $[13,14]$ :

$$
\frac{\partial \mathcal{L}}{\partial \mathcal{F}}=-\frac{g^{2}}{\bar{g}^{2}(t)}, \quad \frac{d \bar{g}}{d t}=\beta(\bar{g}), \quad t=\frac{1}{2} \ln \left(2 g^{2} \mathcal{F} / \mu^{4}\right) .
$$

The derivative (2.1) of the effective Lagrangian has transparent expression in terms of the effective coupling constant and allows to obtain the effective Lagrangian by integration over $\mathcal{F}$ in all order of the perturbative expansion:

$$
\mathcal{L}(\mathcal{F})=-\mu^{4} \int \frac{e^{2 t}}{\bar{g}^{2}(t)} d t, \quad t=\frac{1}{2} \ln \left(2 g^{2} \mathcal{F} / \mu^{4}\right),
$$

and find out the expressions for the physical quantities beyond the one-loop approximation. One can calculate different observables of physical interest that will include the effective energy momentum tensor, vacuum energy density, the magnetic permeability, the effective coupling constants and their behaviour as a function of the external fields. In particular, the vacuum magnetic permeability introduced in (1.6) will take the following form [11]:

$$
\mu_{v a c}=\frac{g^{2}}{\bar{g}^{2}(t)}, \quad \mathcal{G}=0
$$


and the energy momentum tensor (1.9) can expressed in the form:

$$
T_{\mu \nu}=-\left(G_{\mu \lambda} G_{\nu \lambda}-g_{\mu \nu} \frac{1}{4} G_{\lambda \rho}^{2}\right) \frac{g^{2}}{\bar{g}^{2}(t)}+g_{\mu \nu}\left(\int \frac{e^{2 t}}{\bar{g}^{2}(t)} d t-\frac{1}{2} \frac{e^{2 t}}{\bar{g}^{2}(t)}\right) \mu^{4} .
$$

The vacuum energy density in terms of the trace $T_{\mu \mu}$ is:

$$
\epsilon=T_{00}=\frac{\overrightarrow{\mathcal{H}}_{a}^{2}}{2} \frac{g^{2}}{\bar{g}^{2}(t)}+\frac{1}{4} T_{\mu \mu}, \quad \mathcal{G}=0,
$$

where the trace of the energy momentum tensor $T_{\mu \mu}$ is given by the following expression:

$$
T_{\mu \mu}=4 \mu^{4} \int \frac{e^{2 t} \beta(\bar{g}(t))}{\bar{g}(t)^{3}} d t, \quad t=\frac{1}{2} \ln \left(2 g^{2} \mathcal{F} / \mu^{4}\right) .
$$

The last formula provides all-loop expression for the conformal anomaly in gauge field theories $^{2}$. As far as the beta function $\bar{\beta}(g)$ has no zeros, is negative analytical function of the coupling constant and $\int_{g}^{\infty} \frac{d g}{\beta(g)}<\infty$, the minimum of the energy density curve is defined by the extremum, where the derivative (2.1) vanishes. It follows that the value of the chromomagnetic condensate is [13]

$$
\left\langle 2 g^{2} \mathcal{F}\right\rangle_{v a c}=\mu^{4} \exp \left(2 \int_{g(\mu)}^{\infty} \frac{d g}{\beta(g)}\right) .
$$

Let us analyse the behaviour of the effective coupling constant. The Callan-Symanzik beta function can be calculated by using (2.1) and (1.4):

$$
\beta=\left.\frac{1}{2} g \frac{\partial \mathcal{M}}{\partial t}\right|_{t=0}=-\frac{11 N}{96 \pi^{2}} g^{3}
$$

and the effective coupling constant as a function of the field takes the form

$$
\bar{g}^{2}(\mathcal{F})=\frac{g^{2}}{1+\frac{11 g^{2} N}{96 \pi^{2}} \ln \frac{2 g^{2} \mathcal{F}}{\mu^{4}}},
$$

where we introduced the Casimir operator $C_{2}(G)=N$ for the gauge group $G=S U(N)$. Let us consider the value of the field strength tensor $\mathcal{F}_{0}$ at which the vacuum energy density (1.14) vanishes $\epsilon\left(\mathcal{F}_{0}\right)=0$, as it is shown on Fig.2:

$$
2 g^{2} \mathcal{F}_{0}=\mu^{4} \exp \left(-\frac{96 \pi^{2}}{11 g^{2} N}+1\right)=e\left\langle 2 g^{2} \mathcal{F}\right\rangle_{\text {vac }} .
$$

The effective coupling constant (2.9) at this field strength has the value

$$
\bar{g}^{2}\left(\mathcal{F}_{0}\right)=\frac{96 \pi^{2}}{11 N} .
$$

It follows that the effective coupling constant at the intersection point $\mathcal{F}_{0}$ is small:

$$
\bar{g}^{2}\left(\mathcal{F}_{0}\right)=\frac{96 \pi^{2}}{11 N} \ll 1 \quad \text { if } \quad N \gg \frac{96 \pi^{2}}{11} .
$$

\footnotetext{
${ }^{2}$ If one considers the approximation in which $\bar{g}(t)$ is field independent $\bar{g}(t) \equiv g$ then (2.6) will reduce to the one given in literature $[55,56,57,58]$.
} 
The energy density curve $\epsilon(\mathcal{F})$ (1.14) intersects the horizontal zero energy line at the nonzero angle $\theta$ (see Fig.2):

$$
\tan \theta=\frac{11 g^{2} N}{96 \pi^{2}}>0 .
$$

This means that i) the true vacuum state is below the perturbative vacuum and that ii) there is a nonzero chromomagnetic field in the vacuum. Now the question is, how far into the infrared region one can continue the energy density curve by using the one-loop result? Let us consider the fields which are approaching the infrared pole. This can be done, in particular, by using the following parametrisation:

$$
\mathcal{F}_{n}=e^{1-n}\langle\mathcal{F}\rangle_{\text {vac }}
$$

where the parameter $n$ is less than one, and we have $\mathcal{F}_{n} \rightarrow\langle\mathcal{F}\rangle_{\text {vac }}$ when $n$ tends to unity from below. At these fields values the effective coupling constant (2.9) tends to zero:

$$
\bar{g}^{2}\left(\mathcal{F}_{n}\right)=\frac{96 \pi^{2}}{11 N(1-n)} \rightarrow 0
$$

if the product $N(1-n) \rightarrow \infty$ is large and the t'Hooft coupling constant $g^{2} N=\lambda$ is fixed and small. It follows then that the effective coupling constant can be made small in order to justify the use of the one-loop result and the behaviour of energy density curve which is continuously extended infinitesimally close to the value of the vacuum field $\langle\mathcal{F}\rangle_{\text {vac }}$, as it is shown on Fig.2. Let us analyse how the field at the intersection point (2.10) and the effective coupling constant (2.11) are changing when we include the two-loop contribution. The two-loop ${ }^{3}$ effective Lagrangian has the form [11]

$$
\mathcal{L}=-\mathcal{F}-\left(\frac{11}{6(4 \pi)^{2}} g^{2} N+\frac{34}{6(4 \pi)^{4}}\left(g^{2} N\right)^{2}\right) \mathcal{F}\left(\ln \frac{2 g^{2} \mathcal{F}}{\mu^{4}}-1\right)
$$

The field at the intersection point (2.10) is shifted by an exponentially small correction

$$
2 g^{2} \mathcal{F}_{0}^{\prime}=\exp \left(-\frac{96 \pi^{2}}{11 \lambda} \cdot \frac{1}{1+\frac{17}{88 \pi^{2}} \lambda}+1\right) .
$$

At this field the effective coupling constant is smaller by the factor $1 / 1+\frac{17}{88 \pi^{2}} \lambda$

$$
\bar{g}^{2}\left(\mathcal{F}_{0}^{\prime}\right)=\frac{96 \pi^{2}}{11 N} \cdot \frac{1}{1+\frac{17}{88 \pi^{2}} \lambda} \ll 1,
$$

and the inequality (2.18) is fulfilled at smaller values of $N$ than in the first approximation (2.12). The chromomagnetic condensate in the two-loop approximation will take the following form:

$$
\left\langle 2 g^{2} \mathcal{F}\right\rangle_{v a c}=\mu^{4} \exp \left(-\frac{1}{\beta_{1} g^{2}}\left[1-\frac{\beta_{2} g^{2}}{\beta_{1}} \ln \left(1+\frac{\beta_{1}}{\beta_{2} g^{2}}\right)\right]\right) .
$$

\footnotetext{
${ }^{3}$ The beta function coefficients $\bar{\beta}=-\beta_{1} g^{3}-\beta_{2} g^{5}+.$. are given by $\beta_{1}=\frac{11 N}{6(4 \pi)^{2}}$ and $\beta_{2}=\frac{34 N^{2}}{6(4 \pi)^{4}}[60,61]$.
} 
It is interesting to know if the energy density curve is a continuous function of the field strength $\mathcal{F}$ in the infrared region $\left[0,\langle\mathcal{F}\rangle_{v a c}\right]$, which is outside of the validity of the perturbative calculations, and if the energy density curve is a convex function for all values of fields: from ultraviolet region down to infrared region. A non-perturbative functional method developed by Zwanziger in [42] is answering to these questions affirmatively. It seems that further development of his approach can shed even more light to the behaviour of the effective Lagrangian in the infrared region $\left[0,\langle\mathcal{F}\rangle_{\text {vac }}\right]$.

Let us see what can be obtained in this respect analysing the renormalisation group results. We already obtained the first derivative of the energy density curve (2.1),(2.4) and can calculate its second derivative as well:

$$
\frac{\partial \epsilon}{\partial \mathcal{F}}=\frac{g^{2}}{\bar{g}^{2}}, \quad \mathcal{F} \frac{\partial^{2} \epsilon}{\partial \mathcal{F}^{2}}=-g^{2} \frac{\beta(\bar{g})}{\bar{g}^{3}} .
$$

Thus the convexity of the energy density curve is defined by its second derivative which depends on the sign of the beta function. In QCD, in the perturbative regime the $\beta(\bar{g})$ is negative and the second derivative in (2.20) is positive:

$$
\mathcal{F} \frac{\partial^{2} \epsilon}{\partial \mathcal{F}^{2}}=\frac{11}{6(4 \pi)^{2}} g^{2} N+\frac{34}{6(4 \pi)^{4}}\left(g^{2} N\right)^{2}+\ldots
$$

and the energy density curve is convex (see Fig.2). In QED the overall sign is negative and the energy density curve is concave (see Fig.1).

In summary one can conclude that any non-perturbative information about the ratio $\beta(\bar{g}) / \bar{g}^{3}$ can be translated into the information about property of the energy density curve $\epsilon(\mathcal{F})$. As far as the beta function $\beta(g)$ has no zeros, is a negative analytical function of the coupling constant and $\int_{g}^{\infty} \frac{d g}{\beta(g)}<\infty$, then it follows from (2.20) that the energy density curve is convex and that the minimum of the energy density curve $\epsilon(\mathcal{F})$ is defined by the extremum where its first derivative vanishes.

In this presentation we discussed the phenomena of the YM condensation investigating the effective action $\Gamma[\langle A\rangle]$, a functional which depends on the vacuum expectation value of the gauge field operators $A_{\mu}^{a}(x) \equiv\left\langle 0\left|\mathcal{A}_{\mu}^{a}(x)\right| 0\right\rangle$, as it was defined in (1.1). The condensate is of the chromomagnetic type and has a physical value proportional to the $\Lambda_{Q C D}^{4}$ which is of the order of few hundred $\mathrm{MeV}^{4}$

$$
\left\langle 2 g^{2} \mathcal{F}\right\rangle_{v a c}=\left\langle\frac{g^{2}}{2} G_{\mu \nu}^{2}\right\rangle_{v a c}=\left\langle g^{2}\left(\overrightarrow{\mathcal{H}}_{a}^{2}-\overrightarrow{\mathcal{E}}_{a}^{2}\right)\right\rangle_{v a c}=\mu^{4} \exp \left(2 \int_{g}^{\infty} \frac{d g}{\bar{\beta}(g)}\right)=\Lambda_{Q C D}^{4}>0
$$

or in terms of the strong coupling constant

$$
\left\langle\frac{\alpha_{s}}{\pi} G_{\mu \nu}^{2}\right\rangle_{v a c}=\left\langle\frac{g^{2}}{4 \pi^{2}} G_{\mu \nu}^{2}\right\rangle_{v a c}=\frac{\Lambda_{Q C D}^{4}}{2 \pi^{2}} .
$$

It should be stressed that in our investigation we were studying the effective action $\Gamma[A]$ (1.1), which depends on the VEV of the gauge field operator $\left\langle 0\left|\mathcal{A}_{\mu}^{a}(x)\right| 0\right\rangle \equiv A_{\mu}^{a}(x)$. The effective action is well defined functional, renormalisable in all order of the loop expansion and fulfils the renormalisation group equation. Its first variational derivative provides a complete quantum mechanical equations of motion $\delta \Gamma[A] / \delta A_{\mu}^{a}(x)=0$. 


\section{Conclusion}

The short overview of the publications devoted to the chromomagnetic gluon condensation and QCD vacuum are given below. The confinement problem from the point of view of the QCD vacuum and chromomagnetic gluon condensate was considered in the articles of Mandelshatam [69, 70, 71], Nambu [72], Adler and Piran [74] and Nielsen and Olesen [34]. The thermodynamics of the Yang-Mills gas by Linde [73]. The publication on generation of galactic magnetic field due to the condensation of vector field was considered in [67]. The induced gravity was considered by Adler [59]. The magnetostatics was considered in [68]. The phenomenology of hadrons and the properties of the QCD vacuum by Shuryak [66]. The mechanism of dynamical supersymmetry breaking and string compactification to four dimension due to the properties of the non-Abelian effective action was suggested by Veneziano and Taylor [62]. The dynamical mass generation in QCD and glueballs spectrum by Cornwall [75, 76]. The string-like solution in YM theory were considered in [63, 64, 65]. Knot-like string solutions in YM theory stabilised in the presence of the chromomagnetic condensate by Faddeev and Niemi [77, 78] and the monopole condensation by Cho [79]. A complementary approach for the construction of effective actions at different scales, the Wilsonian effective actions, was developed by Reuter and Wetterich in $[80,81,82,83]$. The effective action in generalised YM theory was considered in [97].

Alternative approach for the investigation of the Yang-Mills condensates was suggested in $[84,85,86,87,88]$ by performing the Monte Carlo lattice simulations. The lattice formulation is offering a non-perturbative regularisation of the YM theory and in principle allows to measure different condensates. The aims was to extract the VEV of the composite operator:

$$
\left\langle 0\left|\frac{\alpha_{s}}{\pi} \mathcal{G}_{\mu \nu} \mathcal{G}_{\mu \nu}\right| 0\right\rangle
$$

In perturbation theory this VEV is represented by the vacuum diagrams without external particles, these diagrams are diverging as the fourth power of the cutoff and in a standard renormalisation scheme are set equal to zero. As it was stressed in references $[84,85,86,87$, 88], the main difficulty in measuring the condensates of the type (3.1) lies in the necessity to subtract the dominant and diverging perturbative contribution and then extract the exponentially falling non-perturbative term which is the only one of interest from the point of view of continuum theory. The evaluation of the VEV requires the calculation of the expression: $\lim _{a \rightarrow 0} \frac{C}{a^{4}}\langle 0|(1-P)| 0\rangle_{\text {meas }}-\langle 0|(1-P)| 0\rangle_{\text {pert }}=\left\langle 0\left|\frac{\alpha_{s}}{\pi} G_{\mu \nu} G_{\mu \nu}\right| 0\right\rangle+\ldots$, where $P$ is the plaquette operator and dots denote the operators of higher dimension. The perturbative VEV is represented by a diverging series [89, 90, 91]: $\langle 0|(1-P)| 0\rangle_{\text {pert }}=$ $\sum_{n=0}^{\infty} c_{n} \alpha_{s}^{n}$. It was demonstrated in $[84,85,86,87,88]$, that the separation of perturbative and non-perturbative contributions has arbitrariness which makes the determination of the composite operator condensate (3.1) ambiguous. In our investigation we were studying a different observable, the effective action $\Gamma[A]$, which depends on the VEV of the gauge field theory operator $A \equiv\langle 0|\mathcal{A}| 0\rangle$. The effective action (1.1) is well defined functional renormalisable in all order of the perturbative loop expansion, fulfils the renormalisation group equations and providing the exact quantum mechanical field equations in terms of its first functional derivative. 
The phenomena of chromomagnetic gluon condensation [13] initiated series of publications by the ITEP group where they used the gluon condensate to improve their perturbative sum rule equations $[92,93]^{4}$. The determination of the gluon condensate numerical value from hadronic $\tau$ decay data and from the charmonium sum rules is reviewed in [94]. The values of the condensate, extracted from QCD sum rules from experimental data, are given in Table 1 [94]. The separation of perturbative and non-perturbative contributions has arbitrariness [93] as it was pointed out by Ioffe [94, 95, 96]. The sum rule equations are defined in terms of the operator product expansion of currents that includes the VEVs of composite operators of the type $\left\langle 0\left|\mathcal{G}_{\mu \nu}^{2}\right| 0\right\rangle$ [94]. In the actual calculations of these VEVs terms, the quark and gluon propagators $\Delta(x, y, A)$ in the background field [12] of the gluon condensate $A \equiv\langle 0|\mathcal{A}| 0\rangle[13]$ are used [93, 94], that yields into the quantity $G_{\mu \nu}^{2}(A)$ instead of $\left\langle 0\left|\mathcal{G}_{\mu \nu}^{2}\right| 0\right\rangle$. This has resulted in an apparent theoretical inconsistency of the sum rule equations and echoes the remarks made above concerning the VEV of composite operator $\left\langle 0\left|\mathcal{G}_{\mu \nu}^{2}\right| 0\right\rangle$ and arbitrariness in separation of perturbative and non-perturbative contributions.

\section{References}

[1] F. Sauter, Uber das Verhalten eines Elektrons im homogenen elektrischen Feld nach der relativistischen Theorie Diracs, Z. Phys. 69 (1931) 742. doi:10.1007/BF01339461

[2] W. Heisenberg, Bemerkungen zur Diracschen Theorie des Positrons, Z. Phys. 90 (1934) 209

[3] H. Euler and B. Kockel, Über die Streuung von Licht an Licht nach der Diracschen Theorie, Naturwiss. 23 (1935) 246.

[4] W. Heisenberg and H. Euler, Consequences of Dirac's theory of positrons, Z. Phys. 98 (1936) 714 .

[5] J. S. Schwinger, On gauge invariance and vacuum polarization, Phys. Rev. 82 (1951) 664. doi:10.1103/PhysRev.82.664

[6] S. R. Coleman and E. J. Weinberg, Radiative Corrections as the Origin of Spontaneous Symmetry Breaking, Phys. Rev. D 7 (1973) 1888. doi:10.1103/PhysRevD.7.1888

[7] V. S. Vanyashin and M. V. Terentev, The Vacuum Polarization of a Charged Vector Field, Zh. Eksp. Teor. Fiz. 48 (1965) no.2, 565 [Sov. Phys. JETP 21 (1965) no.2, 375].

[8] V. V. Skalozub, The Vacuum Polarization of the Charged Vector Field in the Renormalized Theory, Yad. Fiz. 21 (1975) 1337.

[9] M. R. Brown and M. J. Duff, Exact Results for Effective Lagrangians, Phys. Rev. D 11 (1975) 2124. doi:10.1103/PhysRevD.11.2124

[10] M. J. Duff and M. Ramon-Medrano, On the Effective Lagrangian for the Yang-Mills Field, Phys. Rev. D 12 (1975) 3357. doi:10.1103/PhysRevD.12.3357

\footnotetext{
${ }^{4}$ In 1977 the author gave a theoretical seminar on the chromomagnetic gluon condensation [13] in ITEP. At end of the seminar one of the participants, Victor Novikov, on our way back to the metro station by tram, remarked to the author that the theoretical prediction of the chromomagnetic condensate presented at the seminar [13] can be crucial in improving the naive sum rule equations published earlier in [92] by introducing the chromomagnetic condensate in the form of power corrections. A year later, the proposal was realised in [93].
} 
[11] G. K. Savvidy, Vacuum Polarisation by Intensive Gauge Fields, PhD 1977, http://www.inp.demokritos.gr/ savvidy/phd.pdf

[12] I. A. Batalin, S. G. Matinyan and G. K. Savvidy, Vacuum Polarization by a Source-Free Gauge Field, Sov. J. Nucl. Phys. 26 (1977) 214 [Yad. Fiz. 26 (1977) 407].

[13] G. K. Savvidy, Infrared Instability of the Vacuum State of Gauge Theories and Asymptotic Freedom, Phys. Lett. 71B (1977) 133. doi:10.1016/0370-2693(77)90759-6

[14] S. G. Matinyan and G. K. Savvidy, Vacuum Polarization Induced by the Intense Gauge Field, Nucl. Phys. B 134 (1978) 539. doi:10.1016/0550-3213(78)90463-7

[15] G. Savvidy, From Heisenberg-Euler Lagrangian to the discovery of chromomagnetic gluon condensation, Eur. Phys. J. C 80 (2020) no.2, 165 doi:10.1140/epjc/s10052-020-7711-6 [arXiv:1910.00654 [hep-th]].

[16] W. Pauli, Über Gasentartung und Paramagnetizmus, Zs. Phys. 41 (1927) 81

[17] L. Landau, Diamagnetism of Metals, Zs. Phys. 64 (1930) 629

[18] Y. B. Zel'dovich, The Cosmological constant and the theory of elementary particles, Sov. Phys. Usp. 11 (1968) 381 [Usp. Fiz. Nauk 95 (1968) 209]. http://dx.doi.org/10.1070/PU1968v011n03ABEH003927; JETP Lett. 6 (1967) 316

[19] S. Weinberg, The Cosmological constant problem, Rev. Mod. Phys. 61 (1989) 1-23

[20] A. D. Linde, Is the cosmological constant really a constant?, JETP Lett. 19 (1974) 183 [Pisma Zh. Eksp. Teor. Fiz. 19 (1974) 320].

[21] G. Baseyan, S. Matinyan and G. Savvidy, Nonlinear plane waves in the massless Yang-Mills theory, Pisma Zh. Eksp. Teor. Fiz. 29 (1979) 641-644

[22] S. Matinyan, G. Savvidy and N. Ter-Arutyunyan-Savvidi, Classical Yang-Mills mechanics. Nonlinear colour oscillations, Zh. Eksp. Teor. Fiz. 80 (1980) 830-838

[23] G. Savvidy, The Yang-Mills classical mechanics as a Kolmogorov system, Phys. Lett. 130B (1983) 303-307 ; The Yang-Mills quantum mechanics, Phys. Lett. 159B (1985) 325-329

[24] G. Savvidy, Classical and Quantum mechanics of non-Abelian gauge fields, Nucl. Phys. 246 (1984) 302-334

[25] L. D. Landau, A. A. Abeikosov and I. M. Halatnikov, Asymptotic Expression for the Photon Green Function in Quantum Electrodynamics Dokl. Akad. Nauk SSSR 95 (1954) 1177.

[26] B. L. Ioffe, Bez retushi, [Without Retouching] (in Russian), Phasis. Printing House "Nauka" Moscow 2004, 17-19.

[27] H. B. Nielsen, Approximate QCD Lower Bound for the Bag Constant B, Phys. Lett. 80B (1978) 133. doi:10.1016/0370-2693(78)90326-X

[28] N. K. Nielsen and P. Olesen, An Unstable Yang-Mills Field Mode, Nucl. Phys. B 144 (1978) 376. doi:10.1016/0550-3213(78)90377-2

[29] V. V. Skalozub, On Restoration of Spontaneously Broken Symmetry in Magnetic Field, Yad. Fiz. 28 (1978) 228.

[30] J. Ambjorn, N. K. Nielsen and P. Olesen, A Hidden Higgs Lagrangian in QCD, Nucl. Phys. B 152 (1979) 75. doi:10.1016/0550-3213(79)90080-4 
[31] H. B. Nielsen and M. Ninomiya, A Bound on Bag Constant and Nielsen-Olesen Unstable Mode in QCD, Nucl. Phys. B 156 (1979) 1. doi:10.1016/0550-3213(79)90490-5

[32] H. B. Nielsen and P. Olesen, A Quantum Liquid Model for the QCD Vacuum: Gauge and Rotational Invariance of Domained and Quantized Homogeneous Color Fields, Nucl. Phys. B 160 (1979) 380. doi:10.1016/0550-3213(79)90065-8

[33] H. B. Nielsen and M. Ninomiya, Instanton Correction to Some Vacuum Energy Densities and the Bag Constant, Nucl. Phys. B 163 (1980) 57. doi:10.1016/0550-3213(80)90390-9

[34] H. B. Nielsen and P. Olesen, Quark Confinement In A Random Color Magnetic Ether, NBI-HE-79-45.

[35] J. Ambjorn and P. Olesen, On the Formation of a Random Color Magnetic Quantum Liquid in QCD, Nucl. Phys. B 170 (1980) 60. doi:10.1016/0550-3213(80)90476-9

[36] J. Ambjorn and P. Olesen, A Color Magnetic Vortex Condensate in QCD, Nucl. Phys. B 170 (1980) 265. doi:10.1016/0550-3213(80)90150-9

[37] V. V. Skalozub, Nonabelian Gauge Theories In External Electromagnetic Field. (in Russian), Yad. Fiz. 31 (1980) 798.

[38] H. Leutwyler, Vacuum Fluctuations Surrounding Soft Gluon Fields, Phys. Lett. 96B (1980) 154. doi:10.1016/0370-2693(80)90234-8

[39] H. Leutwyler, Constant Gauge Fields and their Quantum Fluctuations, Nucl. Phys. B 179 (1981) 129. doi:10.1016/0550-3213(81)90252-2

[40] C. A. Flory, A Selfdual Gauge Field, Its Quantum Fluctuations, and Interacting Fermions, Phys. Rev. D 28 (1983) 1425. doi:10.1103/PhysRevD.28.1425

[41] W. Dittrich and M. Reuter, Effective QCD Lagrangian With Zeta Function Regularization, Phys. Lett. 128B (1983) 321. doi:10.1016/0370-2693(83)90268-X

[42] D. Zwanziger, Nonperturbative Modification of the Faddeev-popov Formula and Banishment of the Naive Vacuum, Nucl. Phys. B 209 (1982) 336. doi:10.1016/0550-3213(82)90260-7

[43] C. A. Flory, Covariant Constant Chromomagnetic Fields And Elimination Of The One Loop Instabilities, Preprint, SLAC-PUB-3244, http://www-

public.slac.stanford.edu/sciDoc/docMeta.aspx?slacPubNumber=SLAC-PUB-3244; https://lib-extopc.kek.jp/preprints/PDF/1983/8312/8312331.pdf

[44] D. Kay. Unstable modes, zero modes, and phase transitions in QCD, Ph.D Thesis, Simon Fraser University, August 1985.

[45] A. Yildiz and P. H. Cox, Vacuum Behavior in Quantum Chromodynamics, Phys. Rev. D 21 (1980) 1095. doi:10.1103/PhysRevD.21.1095

[46] G. V. Dunne, Heisenberg-Euler effective Lagrangians: Basics and extensions, hep-th/0406216.

[47] G. L. Pimentel, A. M. Polyakov and G. M. Tarnopolsky, Vacua on the Brink of Decay, Rev. Math. Phys. 30 (2018) no.07, 1840013 doi:10.1142/S0129055X18400135, [arXiv:1803.09168 [hep-th]].

[48] A. G. Riess et al. [Supernova Search Team], Observational evidence from supernovae for an accelerating universe and a cosmological constant, Astron. J. 116 (1998) 1009 doi:10.1086/300499 [astro-ph/9805201]. 
[49] J. L. Tonry et al. [Supernova Search Team], Cosmological results from high-z supernovae, Astrophys. J. 594 (2003) 1 doi:10.1086/376865 [astro-ph/0305008].

[50] S. Perlmutter et al. [Supernova Cosmology Project Collaboration], Measurements of Omega and Lambda from 42 high redshift supernovae, Astrophys. J. 517 (1999) 565 doi:10.1086/307221 [astro-ph/9812133].

[51] M. Betoule et al. [SDSS Collaboration], Improved cosmological constraints from a joint analysis of the SDSS-II and SNLS supernova samples, Astron. Astrophys. 568 (2014) A22 doi:10.1051/0004-6361/201423413 [arXiv:1401.4064 [astro-ph.CO]].

[52] R. Adam et al. [Planck Collaboration], Planck 2015 results. I. Overview of products and scientific results, Astron. Astrophys. 594 (2016) A1 doi:10.1051/0004-6361/201527101 [arXiv:1502.01582 [astro-ph.CO]].

[53] N. Aghanim et al. [Planck Collaboration], Planck 2018 results. VI. Cosmological parameters, arXiv:1807.06209 [astro-ph.CO].

[54] R. Pasechnik, G. Prokhorov and O. Teryaev, Mirror QCD and Cosmological Constant, Universe 3 (2017) no.2, 43 doi:10.3390/universe3020043 [arXiv:1609.09249 [hep-ph]].

[55] S. L. Adler, J. C. Collins and A. Duncan, Energy-momentum-tensor trace anomaly in spin 1/2 QED, Phys. Rev. D 15 (1977) 1712.

J. C. Collins, A. Duncan and S. D. Joglekar, Trace And Dilatation Anomalies In Gauge Theories, Phys. Rev. D 16 (1977) 438.

[56] N.K. Nielsen, The energy-momentum tensor in a non-Abelian quark gluon theory, Nucl.Phys. B 120 (1977) 212.

[57] P. Minkowski, On the anomalous divergence of the dilatation current in gauge theories, Bern preprint (1976).

[58] M. J. Duff, Observations on Conformal Anomalies, Nucl. Phys. B 125 (1977) 334. doi:10.1016/0550-3213(77)90410-2

[59] S. L. Adler, Einstein Gravity as a Symmetry-Breaking Effect in Quantum Field Theory, Rev. Mod. Phys. 54 (1982) 729 Erratum: [Rev. Mod. Phys. 55 (1983) 837]. doi:10.1103/RevModPhys.54.729

[60] D. R. T. Jones, Two Loop Diagrams in Yang-Mills Theory, Nucl. Phys. B 75 (1974) 531. doi:10.1016/0550-3213(74)90093-5

[61] W. E. Caswell, Asymptotic Behavior of Nonabelian Gauge Theories to Two Loop Order, Phys. Rev. Lett. 33 (1974) 244. doi:10.1103/PhysRevLett.33.244

[62] T. R. Taylor and G. Veneziano, Strings and D=4, Phys. Lett. B 212 (1988) 147. doi:10.1016/0370-2693(88)90515-1

[63] N. K. Nielsen and P. Olesen, Electric Vortex Lines From the Yang-Mills Theory, Phys. Lett. 79B (1978) 304. doi:10.1016/0370-2693(78)90249-6

[64] H. B. Nielsen and P. Olesen, Vortex Line Models for Dual Strings, Nucl. Phys. B 61 (1973) 45. doi:10.1016/0550-3213(73)90350-7

[65] C. A. Flory, Stability Properties Of An Abelianized Chromoelectric Flux Tube, Phys. Rev. D 29 (1984) 722. doi:10.1103/PhysRevD.29.722 
[66] E. V. Shuryak, Theory and phenomenology of the QCD vacuum, Phys. Rept. 115 (1984) 151. doi:10.1016/0370-1573(84)90037-1

[67] K. Enqvist and P. Olesen, Ferromagnetic vacuum and galactic magnetic fields, Phys. Lett. B 329 (1994) 195; doi:10.1016/0370-2693(94)90760-9 [hep-ph/9402295].

[68] H. Pagels and E. Tomboulis, Vacuum of the Quantum Yang-Mills Theory and Magnetostatics, Nucl. Phys. B 143 (1978) 485. doi:10.1016/0550-3213(78)90065-2

[69] S. Mandelstam, Approximation Scheme for QCD, Phys. Rev. D 20 (1979) 3223. doi:10.1103/PhysRevD.20.3223

[70] S. Mandelstam, Review of recent results on QCD and confinement, UCB-PTH-79-9.

[71] S. Mandelstam, General Introduction To Confinement, Phys. Rept. 67 (1980) 109. doi:10.1016/0370-1573(80)90083-6;

[72] Y. Nambu, Effective Abelian Gauge Fields, Phys. Lett. 102B (1981) 149. doi:10.1016/0370-2693(81)91051-0

[73] A. D. Linde, Infrared Problem in Thermodynamics of the Yang-Mills Gas, Phys. Lett. 96B (1980) 289. doi:10.1016/0370-2693(80)90769-8

[74] S. L. Adler and T. Piran, Relaxation Methods for Gauge Field Equilibrium Equations, Rev. Mod. Phys. 56 (1984) 1. doi:10.1103/RevModPhys.56.1

[75] J. M. Cornwall, Dynamical Mass Generation in Continuum QCD, Phys. Rev. D 26 (1982) 1453. doi:10.1103/PhysRevD.26.1453

[76] J. M. Cornwall and A. Soni, Couplings Of Low Lying Glueballs To Light Quarks, Gluons, And Hadrons, Phys. Rev. D 29 (1984) 1424. doi:10.1103/PhysRevD.29.1424

[77] L. D. Faddeev and A. J. Niemi, Aspects of Electric magnetic duality in SU(2) Yang-Mills theory, Phys. Lett. B 525 (2002) 195 doi:10.1016/S0370-2693(01)01432-0 [hep-th/0101078].

[78] L. D. Faddeev, Notes on divergences and dimensional transmutation in Yang-Mills theory, Theor. Math. Phys. 148 (2006) 986 [Teor. Mat. Fiz. 148 (2006) 133]. doi:10.1007/s11232-006-0095-4

[79] Y. M. Cho, Monopole condensation and mass gap in SU(3) QCD, Int. J. Mod. Phys. A 29 (2014) 1450013. doi:10.1142/S0217751X14500134

[80] M. Reuter and C. Wetterich, Indications for gluon condensation for nonperturbative flow equations, hep-th/9411227.

[81] M. Reuter and C. Wetterich, Search for the QCD ground state, Phys. Lett. B 334 (1994) 412 doi:10.1016/0370-2693(94)90707-2 [hep-ph/9405300].

[82] M. Reuter and C. Wetterich, Effective average action for gauge theories and exact evolution equations, Nucl. Phys. B 417 (1994) 181. doi:10.1016/0550-3213(94)90543-6

[83] C. Wetterich, Exact evolution equation for the effective potential, Phys. Lett. B 301 (1993) 90 doi:10.1016/0370-2693(93)90726-X [arXiv:1710.05815 [hep-th]].

[84] A. Di Giacomo and G. C. Rossi, Extracting the Vacuum Expectation Value of the Quantity $\alpha \times G^{2} / \pi$ from Gauge Theories on a Lattice, Phys. Lett. 100B (1981) 481 . doi:10.1016/0370-2693(81)90609-2 
[85] J. Kripfganz, Gluon Condensate From SU(2) Lattice Gauge Theory, Phys. Lett. 101B (1981) 169. doi:10.1016/0370-2693(81)90666-3

[86] A. Di Giacomo and G. Paffuti, Precise Determination of Vacuum Expectation Value of $\alpha \times G^{2} / \pi$ From Lattice Gauge Theories, Phys. Lett. 108B (1982) 327 . doi:10.1016/0370-2693(82)91204-7

[87] E. M. Ilgenfritz and M. Muller-Preussker, SU(3) Gluon Condensate From Lattice MC Data, Phys. Lett. 119B (1982) 395. doi:10.1016/0370-2693(82)90698-0

[88] G. S. Bali, C. Bauer and A. Pineda, Model-independent determination of the gluon condensate in four-dimensional SU(3) gauge theory, Phys. Rev. Lett. 113 (2014) 092001 doi:10.1103/PhysRevLett.113.092001 [arXiv:1403.6477 [hep-ph]].

[89] F. J. Dyson, Divergence of perturbation theory in quantum electrodynamics, Phys. Rev. 85 (1952) 631. doi:10.1103/PhysRev.85.631

[90] L. N. Lipatov, Divergence of the Perturbation Theory Series and the Quasiclassical Theory, Sov. Phys. JETP 45 (1977) 216 [Zh. Eksp. Teor. Fiz. 72 (1977) 411].

[91] G. 't Hooft, in Proceedings of the International School of Subnuclear Physics: The Whys of Subnuclear Physics, Erice, 1977, edited by A. Zichichi (Plenum, New York, 1979).

[92] V. A. Novikov, L. B. Okun, M. A. Shifman, A. I. Vainshtein, M. B. Voloshin and V. I. Zakharov, Sum Rules for Charmonium and Charmed Mesons Decay Rates in Quantum Chromodynamics, Phys. Rev. Lett. 38 (1977) 626. doi:10.1103/PhysRevLett.38.791.2

[93] V. I. Zakharov, Gluon condensate and beyond, Int. J. Mod. Phys. A 14 (1999) 4865 doi:10.1142/S0217751X9900230X [hep-ph/9906264].

[94] B. L. Ioffe, V. S. Fadin and L. N. Lipatov, Quantum chromodynamics: Perturbative and nonperturbative aspects, Camb. Monogr. Part. Phys. Nucl. Phys. Cosmol. 30 (2010). doi:10.1017/CBO9780511711817

[95] K. Zyablyuk, Gluon condensate and c quark mass in pseudoscalar sum rules at three loop order, JHEP 0301 (2003) 081; doi:10.1088/1126-6708/2003/01/081 [hep-ph/0210103].

[96] A. Samsonov, Gluon condensate in charmonium sum rules for the axial-vector current, hep-ph/0407199.

[97] G. Savvidy, Generalisation of the Yang-Mills Theory, Int. J. Mod. Phys. A 31 (2016) 1630003. doi:10.1142/S0217751X16300039; Proceedings of the Conference on 60 Years of Yang-Mills Gauge Field Theories. Nanyang Technological University, Singapore, 25 - 28 May 2015. doi:10.1142/9789814725569-0015 